\title{
Dynamic Hypergames for Synthesis of Deceptive Strategies with Temporal Logic Objectives
}

\author{
Lening Li (D), Student Member, IEEE, Haoxiang Ma (D), Student Member, IEEE, Abhishek N. Kulkarni \\ Student Member, IEEE, and Jie Fu (D), Member, IEEE
}

\begin{abstract}
In this paper, we study the use of deception for strategic planning in adversarial environments. We model the interaction between the agent (player 1) and the adversary (player 2) as a two-player concurrent game in which the adversary has incomplete information about the agent's task specification in temporal logic. During the online interaction, the adversary can infer the agent's intention from observations and adapt its strategy so as to prevent the agent from satisfying the task. To plan against such an adaptive opponent, the agent must leverage its knowledge about the adversary's incomplete information to influence the behavior of the opponent, and thereby being deceptive. To synthesize a deceptive strategy, we introduce a class of hypergame models that capture the interaction between the agent and its adversary given asymmetric, incomplete information. A hypergame is a hierarchy of games, perceived differently by the agent and its adversary. We develop the solution concept of this class of hypergames and show that the subjectively rationalizable strategy for the agent is deceptive and maximizes the probability of satisfying the task in temporal logic. This deceptive strategy is obtained by modeling the opponent evolving perception of the interaction and integrating the opponent model into proactive planning. Following the deceptive strategy, the agent chooses actions to influence the game history as well as to manipulate the adversary's perception so that it takes actions that benefit the goal of the agent. We demonstrate the correctness of our deceptive planning algorithm using robot motion planning examples with temporal logic objectives and design a detection mechanism to notify the agent of potential errors in modeling of the adversary's behavior.
\end{abstract}

Index Terms-Hypergame, Markov Decision Processes, Linear Temporal Logic, Deception.

Note to Practitioners-Many security and defense applications employ deception mechanisms for strategic advantages. This work presented a game-theoretic framework for planning deceptive strategies in stochastic environments and shows that the opponent modeling plays a key role in the design of effective deception mechanisms. For applications to cyber-physical security, the practitioners can employ temporal logic for specifying security properties in the system and analyze defense with deception using the proposed methods.

\section{INTRODUCTION}

$\mathbf{P}$ LANNING in adversarial environments is commonly encountered in security and defense applications. When

Lening Li, Haoxiang Ma, and Abhishek N. Kulkarni are with Robotics Engineering Program, and Jie Fu is with Faculty of Electrical and Computer Engineering, with affiliation to Robotics Engineering Program, Worcester Polytechnic Institute, Worcester, MA. 01609. e-mail: 1li4, hma2, ankulkarni, jfu2@wpi.edu.

This material is based upon work supported by the Defense Advanced Research Projects Agency (DARPA) under Agreement No. HR00111990015. Approved for public release; distribution is unlimited. the objective of a planning agent is partially unknown to its adversary, deception becomes inseparable from the strategic planning: With a proper deception mechanism, the adversary can be misled to take actions beneficial to the agent. Scenarios of interest include economics [1], military operations [2], securities in cyber-network [3], [4], [5], [6], robotics, and other cyber-physical systems [7], [8], [9], [10], [11]. This paper investigates deceptive planning by developing solution concepts for a class of concurrent stochastic games with asymmetric information and Boolean payoffs in temporal logic [12]. In this game, the agent (player 1/P1) is to achieve a task specified as a temporal logic formula [13], which expresses desired behaviors including safety, reachability, obligation, and liveness. The asymmetry of information is introduced such that the adversary (player 2/P2) does not know player 1's task formula but is aware of its incomplete information about player 1. The question is, how could player 1 leverage player 2's incomplete information for optimizing task performance?

To address this question, we take a game-theoretic approach. In literature, deceptive planning has employed the solution concepts of games with asymmetrical information including dynamic Bayesian games [5], [14] and hypergames [15], [16]. The authors [5] adopted dynamic Bayesian games to defensive planning in cyber deception, where the type of the opponent, i.e., a legitimate user or an attacker, was the private information. They captured the incomplete information as a probability distribution over a set of the opponents' types and employed Bayesian Nash Equilibrium to design a defensive strategy. Hypergame [17], [18] was characterized by players' misperception of the other players' payoffs or other components of the game (state/action space). In repeated normal-form hypergames, the authors [10] studied how a player's belief of other players' preferences evolves by observing other players' decisions and analyzing the inconsistency in the equilibrium. They developed stealthy deception [19] to restrict the deceiver's actions so as not to contradict the belief of the deceivee. Deception has been also investigated for enhancing security of cyber-physical systems (CPSs). In [20], the authors proposed a deception-as-defense framework to ensure optimal system performance given adversarial inputs in communication and control of continuous systems. The use of deception is to allow the agent with private information to craft the information perceived by adversary in order to control adversary's perception of the state of the system. For the supervisory control of CPSs, the authors [21] developed an algorithm to synthesize an attack strategy with which the attacker modifies sensor readings and thus misleads the 
supervisor to achieve undesirable states in the discrete event systems. It is noted that these deception methods in CPSs are concerned with deceptive information exchange, instead of the payoffs about the game. Besides game-theoretic approaches, deceptive planning was developed in [9], [11] when the deceiver hides its objective from the observer for achieving the goal. However, there is no dynamic interaction between the deceiver and the observer.

A major characteristic in our game model is that players' payoffs are temporal logic formulas, instead of utility functions. In reactive synthesis, games with temporal logic payoffs, also known as omega-regular games, are studied for synthesizing provably correct systems in dynamic environments. Existing solution concepts for omega-regular games studied games with complete information and perfect/partial observations [22], [23], [24], [25]. However, to synthesize deceptive strategy given temporal logic tasks, it is necessary to develop new theory and solution concepts of omega-regular games with asymmetric, incomplete information.

To this end, we propose both a modeling framework and synthesis methods for deceptive planning in a subclass of omega-regular games with hierarchical information pattern. In this subclass of omega-regular games, players' objectives are given by syntactically co-safe LTL (scLTL) formula and safe Linear Temporal Logic (LTL) formulas [26]. We extend hypergame definition to define a class of hierarchical omega-regular hypergames. We develop a solution concept called subjectively rationalizable strategies in hypergame. Based on this solution, two key modules, namely, opponent modeling and proactive planning, are identified for effective deception. Given the common knowledge that a temporal logic formula describes a sequence of temporally extended subgoals, we assume that the adversary can infer the current agent's subgoal based on observations. In opponent modeling, the agent maintains a model of the adversary's subjectively rationalizable behavior strategy and subgoal inference. Using the opponent model, the agent predicts the adversary's subjectively rationalizable strategy and plans proactively to satisfy its temporal logic constraints. The term "proactive" means that the agent predicts how its action will influence the perception of the adversary before taking the action. The effectiveness of the proactive strategy hinges on the matching between the agent's opponent model and the true opponent. We design an online detection algorithm to identify potential errors in the opponent model. We show the effectiveness of the proposed solution concept for deceptive planning using robot motion planning examples with temporal logic objectives.

To summarize, the key contributions of the paper are:

- A modeling framework of hierarchical omega-regular hypergames for adversarial interactions with asymmetric information about the payoffs in temporal logic.

- A solution concept for this class of hypergames.

- A proactive deceptive planning method that integrates opponent modeling, intent inference, and probabilistic planning with temporal logic constraints.

- A detection mechanism to identify at runtime any modeling mismatch that may make the deceptive strategy ineffective.
The remainder of this paper is organized as follows. Section II provides some necessary background on game theory and linear temporal logic. Section III presents the main theory and algorithms for deceptive planning. Section IV presents a case study to demonstrate the effectiveness of the deception. Finally, Section V concludes and discusses future work.

\section{Preliminaries and Problem Formulation}

Notation: We use the notation $\Sigma$ for a finite set of symbols, also known as the alphabet. A sequence of symbols $w=\sigma_{0} \sigma_{1} \ldots \sigma_{n}$ with $\sigma_{i} \in \Sigma$ for any $0 \leq i \leq n$, is called a finite word, and $\Sigma^{*}$ is the set of all finite words that can be generated with alphabet $\Sigma$. The empty word, denoted by $\varepsilon$, is the empty sequence. We denote the set of all $\omega$-regular words as $\Sigma^{\omega}$ obtained by concatenating the elements in $\Sigma$ infinitely many times. The length of a word is denoted by $|w|$ (note that $|\varepsilon|=0)$. We define the set of nonempty finite words $\Sigma^{+}=\Sigma^{*} \backslash\{\varepsilon\}$. Given a finite and discrete set $X$, let $\mathcal{D}(X)$ be the set of all probability distributions over $X$.

\section{A. Omega-regular games}

We consider an adversarial encounter between two players; a controllable player P1 (pronoun "he") and an uncontrollable player P2 (pronoun "she"). Both players choose their moves simultaneously. The dynamics of their interaction can be captured as a transition system with simultaneous moves.

Definition 1 (Two-player Transition System with Simultaneous Moves). A two-player transition system with simultaneous moves is a tuple $T S=\left\langle S, A, P, s_{0}, \mathcal{A P}, L\right\rangle$ consisting of the following components:

- $S$ is the set of states.

- $A=A_{1} \times A_{2}$ is a finite set of actions, where $A_{1}$ is the set of actions that $\mathrm{P} 1$ can perform, and $A_{2}$ is the set of actions that $\mathrm{P} 2$ can perform.

- $P: S \times A \rightarrow \mathcal{D}(S)$ is a probabilistic transition function. At every state $s \in S, \mathrm{P} 1$ chooses an action $a_{1} \in A_{1}$, and $\mathrm{P} 2$ chooses an action $a_{2} \in A_{2}$ simultaneously. Then, a successor state $s^{\prime}$ is determined by the probability distribution $P(\cdot \mid s, a)$, where $a=\left(a_{1}, a_{2}\right) \in A$.

- $s_{0}$ in an initial state.

- $\mathcal{A P}$ is a set of atomic propositions.

- $L: S \rightarrow 2^{\mathcal{A P}}$ is a labeling function. For every state $s \in$ $S$, the label $L(s)$ of the state $s$ represents a set of atomic propositions that are evaluated true at the state $s$.

A path $\rho=s_{0} s_{1} \ldots$ is a state sequence such that for any $i \geq$ 0 , there exists $a \in A, P\left(s_{i+1} \mid s_{i}, a\right)>0$. A path $\rho=s_{0} s_{1} \ldots$ can be mapped to a word in $2^{\mathcal{A P}}, w=L\left(s_{0}\right) L\left(s_{1}\right) \ldots$, which is evaluated against logical formulas. In this paper, unless otherwise noted, we will refer to a two-player transition system with simultaneous moves simply as a transition system.

We use linear temporal logic (LTL) formulas to represent the players' objectives/payoffs in the game. Temporal Logic provides a succinct way to represent temporal goals and constraints. An LTL formula is defined inductively as follows:

$$
\varphi:=\top|\perp| p|\varphi| \neg \varphi\left|\varphi_{1} \wedge \varphi_{2}\right| \bigcirc \varphi \mid \varphi_{1} \mathrm{U} \varphi_{2}
$$


where $T$ and $\perp$ are universally true and false, respectively, $p \in \mathcal{A P}$ is an atomic proposition, and $\bigcirc$ is a temporal operator called the "next" operator. $\bigcirc \varphi$ is evaluated to be true if the formula $\varphi$ becomes true at the next time step. $U$ is a temporal operator called the "until" operator. The formula $\varphi_{1} \mathrm{U} \varphi_{2}$ is true given that $\varphi_{2}$ will be true in some future time steps, and before that $\varphi_{1}$ holds true for every time step.

The operators $\diamond$ (read as eventually) and $\square$ (read as always) are defined using the operator $\mathrm{U}$ as follows: $\diamond \varphi=\top \mathrm{U} \varphi$ and $\square \varphi=\neg \diamond \neg \varphi$. The formula $\diamond \varphi$ is true if $\varphi$ becomes true in some future time. The formula $\square \varphi$ means that $\varphi$ will hold for every time step. Given an LTL formula $\varphi$ and a word $w \in \Sigma^{\omega}$, if the word $w$ satisfies the formula $\varphi$, then we denote $w \models \varphi$. We denote a set of LTL formulas as $\Phi$. For details about the syntax and semantics of LTL, the readers are referred to [27].

In this paper, we restrict the specifications of the players to the class of scLTL [13]. An scLTL formula contains only $\diamond$ and $U$ temporal operators when written in a positive normal form (i.e., the negation operator $\neg$ appears only in front of atomic propositions). The unique property of scLTL formulas is that a word satisfying an scLTL formula $\varphi$ only needs to have a good prefix. That is, given a good prefix $w \in \Sigma^{*}$, the word $w w^{\prime} \models \varphi$ for any $w^{\prime} \in \Sigma^{\omega}$. The set of good prefixes can be compactly represented as the language accepted by a Deterministic Finite Automaton (DFA) defined as follows:

Definition 2 (Deterministic Finite Automaton). Given P1's objective expressed as an scLTL formula $\varphi$, the set of good prefixes of words corresponding to $\varphi$ is accepted by a DFA $\mathcal{A}=\langle Q, \Sigma, \delta, \iota, F\rangle$ with the following components:

- $Q$ is a finite set of states.

- $\Sigma=2^{\mathcal{A P}}$ is a finite set of symbols.

- $\delta: Q \times \Sigma \rightarrow Q$ is a deterministic transition function.

- $\iota \in Q$ is the unique initial state.

- $F \subseteq Q$ is a set of final states.

For an input word $w=\sigma_{0} \sigma_{1} \ldots \in \Sigma^{\omega}$, the DFA generates a sequence of states $q_{0} q_{1} \ldots$ such that $q_{0}=\iota$ and $q_{i+1}=$ $\delta\left(q_{i}, \sigma_{i}\right)$ for any $i \geq 0$. The word $w$ is accepted by the DFA if and only if there exists $k \geq 0$ such that $q_{k} \in F$. The set of words accepted by the DFA $\mathcal{A}$ is called its language. We assume that the DFA is complete - that is, for every state-action pair $(q, \sigma), \delta(q, \sigma)$ is defined. An incomplete DFA can be made complete by adding a sink state $q_{\text {sink }}$ such that $\delta\left(q_{\text {sink }}, \sigma\right)=q_{\text {sink }}$ and directing all undefined transitions to the sink state $q_{\text {sink }}$.

In the interaction between $\mathrm{P} 1$ and $\mathrm{P} 2, \mathrm{P} 1$ aims to maximize the probability of satisfying his objective in temporal logic, say $\varphi_{1}$, over the transition system; P2's objective is to prevent P1 from satisfying his objective. Hence, P2's objective can be denoted by $\varphi_{2}=\neg \varphi_{1}$. Next, we give the definition of zerosum game with Boolean payoffs expressed in temporal logic.

Definition 3 (Omega-Regular Game). Let $\varphi_{1}$ denote the objective of $\mathrm{P} 1$. Then, given a transition system $T S$, a zerosum omega-regular game on a graph is the tuple

$$
\mathcal{G}\left(\varphi_{1}, \neg \varphi_{1}\right)=\left\langle T S, \varphi_{1}, \neg \varphi_{1}\right\rangle
$$

As the objective of $\mathrm{P} 2$ is the logical negation of P1's objective, we omit P2's objective $\neg \varphi_{1}$ from $\mathcal{G}\left(\varphi_{1}, \neg \varphi_{1}\right)$ and simply denote the game as $\mathcal{G}\left(\varphi_{1}\right)$. A path $\rho \in S^{\omega}$ of $T S$ is winning for player $i$ if and only if $L(\rho) \models \varphi_{i}$ - that is, the labeling of that path satisfies player $i$ 's Boolean objective in temporal logic.

A play in the game is constructed as follows: The players start with the initial game state $s_{0}$, simultaneously select a pair of actions $\left(a_{1}, a_{2}\right) \in A$, move to a next state $s_{1}$, and iterate. The game ends when one of the players satisfies its objective. Thus, a play is a sequence of states and actions, denoted by $s_{0} a_{0} s_{1} a_{1} \ldots$ such that $P\left(s_{i+1} \mid s_{i}, a_{i}\right)>0$ for any $i \geq 0$. The set of plays in the game is denoted by Plays. The set of prefixes of plays ending in a state in the game is denoted by PrefPlays. We refer to $h \in$ PrefPlays as a history in the game. Given a history $h$, we denote the projection of $h$ onto the set of states $S$ as $h \iota_{S}$.

A (mixed) strategy $\pi_{i}$ : PrefPlays $\rightarrow \mathcal{D}\left(A_{i}\right)$, for player $i \in\{1,2\}$, is a function that assigns a probability distribution over all actions given a history. Let $\Pi_{i}$ denote the (mixed) strategy space of player $i$. A strategy profile $\left\langle\pi_{1}, \pi_{2}\right\rangle$ is a pair of strategies, one for each player. A strategy profile $\left\langle\pi_{1}, \pi_{2}\right\rangle$ induces a probability measure $\mathbf{P r}^{\left\langle\pi_{1}, \pi_{2}\right\rangle}$ over PrefPlays.

Slightly abusing the notation, given a play $h \in$ Plays, we say that $h \models \varphi$ for an LTL formula $\varphi$ if $L\left(h L_{S}\right) \models \varphi$, that is, the sequence of states labels obtained from the projection of $h$ onto $S$ satisfies the LTL formula $\varphi$.

Given player $i$ 's Boolean objective $\varphi_{i}$, we define the utility function for player $i$ as $u_{i}$ : PrefPlays $(\mathcal{G}) \times \Pi_{i} \times \Pi_{j} \times \Phi \rightarrow \mathbf{R}$, such that for $(i, j) \in\{(1,2),(2,1)\}, u_{i}\left(h, \pi_{i}, \pi_{j}, \varphi_{i}\right)=$ $\operatorname{Pr}^{\left\langle\pi_{i}, \pi_{j}\right\rangle}\left(h h^{\prime}=\varphi_{i}\right)$ is the probability of satisfying the specification $\varphi_{i}$, where $h \in$ PrefPlays is the initial history given that players follow the strategy profile $\left\langle\pi_{i}, \pi_{j}\right\rangle$, and $h^{\prime}$ is the stochastic process induced by strategy profile $\left\langle\pi_{i}, \pi_{j}\right\rangle$ after the initial history $h$.

We present the definition of Nash equilibrium for omegaregular games with complete information as follows.

Definition 4 (Nash equilibrium [28]). A Nash equilibrium of a omega-regular game $\mathcal{G}\left(\varphi_{1}\right)$ is a strategy profile $\left\langle\pi_{1}^{*}, \pi_{2}^{*}\right\rangle$ with the property that for $(i, j) \in\{(1,2),(2,1)\}$ we have

$$
u_{i}\left(h, \pi_{i}^{*}, \pi_{j}^{*}, \varphi_{i}\right) \geq u_{i}\left(h, \pi_{i}, \pi_{j}^{*}, \varphi_{i}\right) .
$$

In zero-sum omega-regular game, the Nash equilibrium $\left\langle\pi_{1}^{*}, \pi_{2}^{*}\right\rangle$ can be obtained as follows:

$$
\left\langle\pi_{1}^{*}, \pi_{2}^{*}\right\rangle=\arg \max _{\pi_{1} \in \Pi_{1}} \min _{\pi_{2} \in \Pi_{2}} \operatorname{Pr}^{\left\langle\pi_{1}, \pi_{2}\right\rangle}\left(h h^{\prime} \models \varphi_{1}\right) .
$$

\section{B. Problem formulation: Planning under Information Asym- metry}

In Def. 3, the game is a common knowledge to both players. We now consider the case when the information about the game (e.g., dynamics, payoffs) between two players is asymmetrical. Specifically, we consider the case when P2 has incomplete information about P1's temporal logic objective.

Assumption 1. The asymmetrical information between players is introduced as follows: 
- P1's objective is $\varphi_{1}$.

- P2 does not know $\varphi_{1}$ but has an initial hypothesis $x_{0}$ and a hypothesis space $X$ about P1's objective.

The assumption describes scenarios commonly encountered in practice, for both cooperative and adversarial interactions. The problem we aim to solve is stated informally as follows:

Problem 1. Given an adversarial encounter between P1 and P2 under information asymmetry as defined by Assumption 1 . how to compute a best-response strategy for P1 that maximizes the probability of satisfying $\varphi_{1}$ while a rational P2 responds optimally given P2's knowledge of the game?

The definition of "best-response" differs from the one for games with symmetric and complete information in equation (1). Next, we introduce the modeling framework of hypergames and present a solution concept for a class of hypergames to solve P1's strategy.

\section{FORMULATING A CLASS OF OMEGA-REGULAR HYPERGAME}

Hypergame, introduced in [18], is capable of modeling strategic interactions when players have asymmetrical information. Intuitively, a hypergame is a game of games, and each game is associated with a player's subjective view of its interaction with other players based on its own information and information about others' subjective views.

\section{A. Static hypergames on graphs}

To start with, given that $\mathrm{P} 2$ has incomplete information, we introduce a hypothesis space for $\mathrm{P} 2$, denoted by $X$. The set $X$ can be discrete and finite. For example, the set $X$ can be a finite set of scLTL formulas that $\mathrm{P} 2$ believes that P1's true objective is one of these. The hypothesis space $X$ can also be continuous. For example, each $x \in X$ is a distribution over a subset of scLTL formulas $\Phi$ so that $x(\varphi)$ is the probability that P2 believes $\varphi$ to be P1's true objective. For the time being, we do not restrict the set $X$.

We can formally introduce a hypergame, which extends the hypergames from normal-form games [18], [29] to omegaregular games.

Definition 5. A static omega-regular hypergame of level-1 is defined as

$$
\mathcal{H} \mathcal{G}^{1}(x)=\left\langle\mathcal{G}\left(\varphi_{1}\right), \mathcal{G}(x)\right\rangle
$$

where $\mathcal{G}\left(\varphi_{1}\right)$ is the game constructed by $\mathrm{P} 1$ given his objective $\varphi_{1}$, and $\mathcal{G}(x)$ is the game constructed by $\mathrm{P} 2$ given her hypothesis $x \in X$. If $\mathrm{P} 1$ is aware of P2's game $\mathcal{G}(x)$, then the resulting hypergame is said to be of level-2 and defined as

$$
\mathcal{H} \mathcal{G}^{2}(x)=\left\langle\mathcal{H} \mathcal{G}^{1}(x), \mathcal{G}(x)\right\rangle .
$$

In a level-2 hypergame, where $\mathcal{H} \mathcal{G}^{1}(x)$ is the level-1 hypergame constructed by $\mathrm{P} 1$ given his objective and his knowledge about the game constructed by $\mathrm{P} 2, \mathrm{P} 1$ computes his strategy by solving the level-1 hypergame $\mathcal{H G}^{1}(x)$ while $\mathrm{P} 2$ computes her strategy by solving the game $\mathcal{G}(x)$.
The game constructed by a player given his/her information and higher-order information is called the player's perceptual game. In level-2 hypergame $\mathcal{H G}^{2}(x), \mathrm{P} 1$ 's perceptual game is $\mathcal{H G}^{1}(x)$ and P2's perceptual game is $\mathcal{G}(x)$.

If the hypothesis $x \in X$ does not change, then we say this game is static. In this static hypergame, players choose actions simultaneously, determined by their respective strategies, and their strategies do not change during their interaction.

Higher levels of hypergames can be defined through a recursive reasoning about higher-order information (e.g., what I know that you know that I know ...). In this scope, we restrict to level-2 hypergames. For simplicity, we refer to level-2 omega-regular hypergames as hypergames in this paper whenever it is clear from the context.

We now discuss the solution concepts of hypergames. Given that different players may have different perceptions (i.e., subjective views) of the utility functions in a hypergame, we denote $u_{i}^{j}$ as the utility function of player $i$ perceived by player $j$. Next, we generalize the related notions of subjective rationalizability and best-response equilibrium in hypergames from normal-form games in [30] to omega-regular hypergames.

Definition 6 (Subjective Rationalizability). Given a level2 hypergame $\mathcal{H G}^{2}(x)=\left\langle\mathcal{H} \mathcal{G}^{1}(x), \mathcal{G}(x)\right\rangle$, strategy $\pi_{i}^{*, 2}$ is subjectively rationalizable (SR) for player 2 if and only if it satisfies, for all $\pi_{i} \in \Pi_{i}$,

$$
u_{i}^{2}\left(h, \pi_{i}^{*, 2}, \pi_{j}^{*, 2}, x\right) \geq u_{i}^{2}\left(h, \pi_{i}, \pi_{j}^{*, 2}, x\right),
$$

where $(i, j) \in\{(1,2),(2,1)\}$. Note that in the case that P2's hypothesis $x$ is a distribution over $\Phi$, then the utility is calculated based on the expectation, that is, $u_{i}^{2}\left(h, \pi_{i}, \pi_{j}, x\right)=$ $\sum_{\varphi \in \Phi} x(\varphi) u_{i}^{2}\left(h, \pi_{i}, \pi_{j}, \varphi\right)$.

The strategy $\pi_{1}^{*, 1}$ is SR for P1 if and only if it satisfies, for all $\pi_{1} \in \Pi_{1}$,

$$
u_{1}^{1}\left(h, \pi_{1}^{*, 1}, \pi_{2}^{*, 2}, \varphi_{1}\right) \geq u_{1}^{1}\left(h, \pi_{1}, \pi_{2}^{*, 2}, \varphi_{1}\right),
$$

where $\pi_{2}^{*, 2}$ is SR for player 2 .

In words, a strategy is called subjectively rationalizable for player $i$ if it is the best response in player $i$ 's perceptual game to a strategy of player $j$, which, for player $j$, is the best response to player $i$ in player $j$ 's subjective view modeled by player $i$ 's perceptual game. A pair of SR strategies $\left\langle\pi_{1}^{*, 1}, \pi_{2}^{*, 2}\right\rangle$ is called the best-response equilibrium of the hypergame $\mathcal{H} \mathcal{G}^{2}(x)$.

In level-2 hypergame, P2's strategy is SR if it is rationalizable in $\mathrm{P} 2$ 's perceptual game $\mathcal{G}(x)$. P1's strategy is SR if it is the best response to P2's SR strategy. However, P1's SR strategy may not be consistent with P2's predicted SR strategy for P1. This inconsistency can be recognized by P2. When P2 notices the mismatch in the perceptual games, her perceptual game may evolve given new information.

\section{B. Dynamic hypergames on graphs}

To characterize P2's evolving perceptual game, we introduce an inference function.

Definition 7 (Inference). Assuming P2 has complete observation on the game plays, a perfect recall inference function 
$\eta: X \times$ PrefPlays $\rightarrow X$ maps a hypothesis $x \in X$ and an observation (a history) $h \in$ PrefPlays to a new hypothesis $x^{\prime}=\eta(x, h) \in X$.

We introduce a transition system of $P 1$ 's level-1 hypergame to capture simultaneously the changes in game states given players' actions and the evolving perceptual game of P2.

Definition 8 (Transition System of P1's Level-1 Hypergame). Given the transition system $T S=\left\langle S, A, P, s_{0}, \mathcal{A P}, L\right\rangle$, the DFA $\mathcal{A}=\langle Q, \Sigma, \delta, \iota, F\rangle$ that corresponds to P1's scLTL specification, and P2's hypotheses space $X$, the transition system of P1's level-1 hypergame is a tuple

$$
\mathcal{H}=\left\langle V, A, \Delta,\left(s_{0}, h_{0}, q_{0}, x_{0}\right), \mathcal{F}\right\rangle,
$$

where the components of hypergame transition system are defined as follows,

- $V=S \times$ PrefPlays $\times Q \times X$ is the set of states. Every state $v=(s, h, q, x) \in V$ has four components:

- $s$ is the state.

- $h \in$ PrefPlays is a history terminating in state $s \in S$.

- $q \in Q$ is the automaton state for keeping track of P1's progress towards satisfying $\varphi_{1}$.

- $x \in X$ represents the hypothesis of $\mathrm{P} 2$ given the history $h$.

- $A$ is the set of joint actions.

- $\Delta: V \times A \rightarrow \mathcal{D}(V)$ is a probabilistic transition function defined as follows. Consider $v=(s, h, q, x)$ and $v^{\prime}=$ $\left(s^{\prime}, h a s^{\prime}, q^{\prime}, x^{\prime}\right)$, where $h a s^{\prime}$ is the history $h$ appended with the new action $a$ and state $s^{\prime}$,

$$
\begin{aligned}
\Delta\left(v^{\prime} \mid v, a\right)= & P\left(s^{\prime} \mid s, a\right) \mathbf{1}\left(\delta\left(q, L\left(s^{\prime}\right)\right)=q^{\prime}\right) \\
& \mathbf{1}\left(\eta\left(x, h a s^{\prime}\right)=x^{\prime}\right),
\end{aligned}
$$

where $\mathbf{1}(E)$ is the indicator function that returns 1 if the statement $E$ is true, and 0 otherwise.

- $\left(s_{0}, h_{0}, q_{0}, x_{0}\right)$ is the initial state that includes the initial state in the transition system $T S$, the current history that consists of the initial state only, i.e., $h_{0}=s_{0}$, $q_{0}=\delta\left(\iota, L\left(s_{0}\right)\right)$, and the initial hypothesis $x_{0}$.

- $\mathcal{F}=S \times$ PrefPlays $\times F \times X$ is the set of final states for P1.

The transition function is understood as follows: Given a history $h$ ending in the current state $s$ and a joint action $a \in A$, the probability of reaching the next state $s^{\prime}$ is determined by $P\left(s^{\prime} \mid s, a\right)$ in the hypergame transition system. Upon reaching $s^{\prime}, \mathrm{P} 2$ updates her hypothesis to $x^{\prime}=\eta\left(x, h a s^{\prime}\right)$ (here we assume the entire history is used for this update). Also, the transition in the specification automaton is triggered to reach state $q^{\prime}=\delta\left(q, L\left(s^{\prime}\right)\right)$ given the labeling of the new state $s^{\prime}$.

Given P2's perceptual game evolving given the history and the inference function, $\mathrm{P} 2$ employs a behaviorally subjectively rationalizable strategy. A strategy is behavioral if it depends on the perceptual game when the action is taken [31]. We define the behaviorally subjectively rationalizable strategy as follows.
Definition 9 (Behaviorally Subjectively Rationalizable (BSR) Strategy). A strategy $\pi_{2}^{B, 2}$ : PrefPlays $\rightarrow \mathcal{D}\left(A_{2}\right)$ is behaviorally subjectively rationalizable (BSR) for P2 if

$$
\pi_{2}^{B, 2}(h)=\pi_{2}^{*, 2}(x, h),
$$

where $x=\eta\left(x_{0}, h\right)$, and $\pi_{2}^{*, 2}(x, \cdot)$ : PrefPlays $\rightarrow \mathcal{D}\left(A_{2}\right)$ is a subjectively rationalizable strategy for $\mathrm{P} 2$ in the hypergame $\mathcal{H G}^{2}(x)$.

Remark 1. When $X$ is finite, the hypergame transition system has a countably infinite set of states. This is because a history can be of a finite but unbounded length. The entire history is maintained as part of the state due to the general definition of the inference mechanism.

\section{Synthesizing Pl's Deceptive Strategy}

Given that P2 uses a BSR strategy, P1 can play deceptively by influencing P2's hypothesis so that P2's actions given her hypothesis can be advantageous for P1. We leverage the hierarchy of reasoning in level-2 hypergames and develop a two-step approach: In the first step, for each state $v=(s, h, q, x)$, we solve P2's BSR strategy using the static hypergame $\mathcal{H G}^{2}(x)$. In the second step, we incorporate P2's BSR strategies into the transition system in Def. 8

This second step is nontrivial as in some games, there are multiple SR strategies for P2 in hypergame $\mathcal{H G}^{2}(x)$. We consider a special case when only one equilibrium exists in the P2's perceptual game $\mathcal{G}(x)$, for each $x \in X$.

We present the solution of P1's strategy for a class of dynamic hypergames that satisfy the following assumptions:

Assumption 2. This subclass of hypergames satisfies the following assumptions:

1) The hypothesis space $X$ is discrete and finite.

2) The inference function $\eta$ has a finite domain. That is, the set of histories are grouped into a finite set of equivalence classes (see the formal definition next).

3) For any $x \in X$, the strategy of $\mathrm{P} 2$ in a Nash equilibrium for a given static hypergame $\mathcal{G}(x)$ is unique.

4) For any $x \in X$, the strategy of $\mathrm{P} 2$ in a Nash equilibrium for a given static hypergame $\mathcal{G}(x)$ is memoryless.

Definition 10 (Inference-equivalent histories). Given an inference function $\eta: X \times$ PrefPlays $\rightarrow X$ and a hypothesis $x$, two histories $h_{1}$ and $h_{2}$ are to be said $(\eta, x)$-equivalent if $\eta\left(x, h_{1}\right)=\eta\left(x, h_{2}\right)$ and for any $h^{\prime} \in(A \times S)^{+}$, $\eta\left(x, h_{1} h^{\prime}\right)=\eta\left(x, h_{2} h^{\prime}\right)$. The set of histories equivalent to $h \in$ PrefPlays given hypothesis $x$ is denoted by $\llbracket h \rrbracket_{x}$. If the equivalence between histories can be defined to be independent of the current hypothesis, that is, for any pair of hypotheses $x, x^{\prime} \in X$, if $h_{1}, h_{2}$ are $(\eta, x)$-equivalent, then $h_{1}, h_{2}$ are also $\left(\eta, x^{\prime}\right)$-equivalent, then we say that the two histories $h_{1}$ and $h_{2}$ are $\eta$-equivalent. The set of histories $\eta$-equivalent to $h \in$ PrefPlays is denoted by $\llbracket h \rrbracket$.

When the set of $\eta$-equivalent (or $(\eta, x)$-equivalent) histories is finite and the set $X$ is finite, the inference $\eta$ has a finite domain and can be equivalently expressed using a Moore machine [32] with inputs histories and outputs hypotheses. 
The set of states in the Moore machine is the set of equivalent classes. We omit the details in constructing the Moore machine and directly incorporate the input-output relations to construct the decision-make problem for solving P1's deceptive strategy.

Definition 11. Under Assumption 2, the deceptive strategy for $\mathrm{P} 1$ in the dynamic hypergame $\mathcal{H}=\left\langle V, A, \Delta,\left(s_{0}, h_{0}, q_{0}, x_{0}\right), \mathcal{F}\right\rangle$ can be obtained by solving the following Markov Decision Process (MDP) with a reachability objective,

$$
\tilde{\mathcal{H}}=\left\langle\tilde{V}, A_{1}, \tilde{\Delta},\left(s_{0}, \llbracket h_{0} \rrbracket_{x_{0}}, q_{0}, x_{0}\right), \mathcal{F}\right\rangle,
$$

where

- $\tilde{V}$ is a finite and discrete set of states. Each state $\tilde{v}=\left(s, \llbracket h \rrbracket_{x}, q, x\right)$ consists of a state $s$, the partition that includes the history classes given the $(\eta, x)$-equivalence relation, a state $q$ in the DFA, and a hypothesis $x$ of $\mathrm{P} 2$.

- $\tilde{\Delta}: \tilde{V} \times A_{1} \rightarrow \mathcal{D}(\tilde{V})$ is defined as follows: For any state $\tilde{v}=\left(s, \llbracket h \rrbracket_{x}, q, x\right)$, if $q \equiv q_{\text {sink }}$ - the absorbing state in the DFA $\mathcal{A}$, then state $\tilde{v}$ is a sink/absorbing state.

Given $\tilde{v}_{1}=\left(s_{1}, \llbracket h_{1} \rrbracket_{x_{1}}, q_{1}, x_{1}\right)$ with $q_{1} \neq q_{\text {sink }}, a_{1} \in A_{1}$, and $\tilde{v}_{2}=\left(s_{2}, \llbracket h_{2} \rrbracket_{x_{2}}, q_{2}, x_{2}\right)$ and $h_{1}\left(a_{1}, a_{2}\right) s_{2} \in \llbracket h_{2} \rrbracket_{x_{2}}$, then

$$
\begin{aligned}
\tilde{\Delta}\left(\tilde{v}_{2} \mid \tilde{v}_{1}, a_{1}\right)= & \sum_{a_{2} \in A_{2}} \pi_{2}^{*, 2}\left(a_{2} \mid x_{1}, s_{1}\right) \\
& P\left(s_{2} \mid s_{1},\left(a_{1}, a_{2}\right)\right) \mathbf{1}\left(\delta\left(q_{1}, L\left(s_{2}\right)\right)=q_{2}\right) .
\end{aligned}
$$

where $\pi_{2}^{*, 2}\left(a_{2} \mid x_{1}, s_{1}\right)$ is the probability of P2 selecting action $a_{2}$ given her current hypothesis $x_{1}$ and the current state $s_{1}$. That is, P2 uses a BSR strategy.

- $\left(s_{0}, \llbracket h_{0} \rrbracket_{x_{0}}, q_{0}, x_{0}\right) \in \tilde{V}$ is the initial state, given $\left(s_{0}, h_{0}, q_{0}, x_{0}\right)$ is the initial state in the transition system $\mathcal{H}$.

- $\tilde{\mathcal{F}}=\left\{\left(s, \llbracket h \rrbracket_{x}, q, x\right) \in \tilde{V} \mid q \in F\right\}$ is the set of final states for P1, where $F$ is the set of final states of DFA $\mathcal{A}$. The states in $\mathcal{F}$ are absorbing.

The size of state space in the MDP is $O(|S| \times N \times|Q| \times$ $|X|)$, where $N$ is the number of $(\eta, x)$-equivalent classes of histories in the game. The size of the action space in the MDP is $O\left(A_{1}\right)$. By construction, if a path $\rho$ in the MDP visits $\tilde{\mathcal{F}}$, then P1 satisfies the LTL formula $\varphi_{1}$. Thus, to maximize the probability of satisfying P1's specification, P1 is to maximize the probability of reaching the set $\tilde{\mathcal{F}}$. We can formulate a Stochastic Shortest Path (SSP) problem assuming each path in the MDP by following a policy will terminate in a sink state. Solving the deceptive strategy for P1 is of time complexity polynomial in the size of state space and action space of the MDP.

An MDP with a reachability objective can be solved using probabilistic model checking algorithms ([33, Chapter 10.1.1],[34]) and existing PRISM toolbox [35]. Given the problem can be of large scale, other approximate dynamic programming solutions of MDP can be used to reduce the dimension of decision variables [36].

Lemma III.1. Under Assumption 2 and assuming P1's knowledge about $\eta$ is correct, then the optimal policy $\pi_{1}^{*}: \tilde{V} \rightarrow$
$\mathcal{D}\left(A_{1}\right)$ in the MDP $\tilde{\mathcal{H}}$ is $\mathrm{P} 1$ 's subjectively rationalizable strategy in the dynamic hypergame given P2's evolving knowledge.

Proof. The construction of $\tilde{\mathcal{H}}$ assumes that P2 follows the behaviorally subjectively rationalizable strategy. Thus, the optimal strategy in $\tilde{\mathcal{H}}$ controls both P1's actions and P2's evolving perceptual game so that P2's actions (predicted by P1) are optimal to P1's objective. Deviating from the strategy cannot gain $\mathrm{P} 1$ a better outcome.

Remark 2. Assumption 2.4) is not necessary. If P2's BSR strategy is represented using a finite-state controller (also known as finite-memory policies), then we can augment the states in the hypergame transition system in Def. 8 with the states in the finite-state controller.

\section{Detecting the Mismatch for Opponent Modeling}

The correctness of P1's BSR strategy hinges on the correctness in the modeling of P2's inference and predicted P2's BSR strategy. In this section, we develop a method to detect if there is any inconsistency between the actual behavior of P2 observed during online interaction and P1's model of P2's behavior used in the MDP $\tilde{\mathcal{H}}$. If the method identifies the inconsistency, then it alerts $\mathrm{P} 1$ that the deceptive policy $\pi_{1}^{*}$ may not be effective.

Consider a finite history $h=s_{0} a_{0} s_{1} a_{1} \ldots s_{n}$, we denote the action pairs for $\mathrm{P} 1$ and $\mathrm{P} 2$ as $a_{i}=\left(a_{1, i}, a_{2, i}\right)$ for any $0 \leq i \leq n-1$. Under the assumption of complete observations, both players can observe the history $h$. By employing the DFA $\mathcal{A}$ corresponding to P1's LTL specification $\varphi_{1}$ and the inference function $\eta$ in the MDP $\tilde{\mathcal{H}}$, we can transform the history $h$ to an augmented state-action sequence denoted by $\tilde{h}=\tilde{v}_{0} a_{0} \tilde{v}_{1} a_{1} \ldots \tilde{v}_{n}$, where $\tilde{v}_{i}=\left(s_{i}, \llbracket h_{i} \rrbracket_{x_{i}}, q_{i}, x_{i}\right)$ and $\tilde{v}_{i+1}=\left(s_{i+1}, \llbracket h_{i+1} \rrbracket_{x_{i+1}}, q_{i+1}, x_{i+1}\right), q_{i+1}=\delta\left(q_{i}, L\left(s_{i+1}\right)\right)$, $h_{i+1}=h_{i} a_{i} s_{i+1}, x_{i+1}=\eta\left(x_{i}, h_{i+1}\right)$. It is worth noting that for a unique history $h$, there exists a unique augmented stateaction sequence $\tilde{h}$ as the transitions in the DFA and inference functions are deterministic, and the entire history up to step $i+1$ is used to compute the $\left(\eta, x_{i+1}\right)$-equivalent histories at step $i+1$. The detection problem reduces to: Given the transition system $T S$, a predicted inference function $\eta$ for $\mathrm{P} 2$ and P2's BSR strategies for a set of hypotheses $X$, how likely is the observation $\tilde{h}$ generated by our predicted model of opponent? If the answer is yes, then there is no mismatch in the model.

We employ likelihood ratio test [37] to answer this question. We have two hypotheses- $H_{0}$ : the data is generated by our predicted model of $\mathrm{P} 2$; and $H_{1}$ : the data is not generated by our prediction. The goal is to test which hypothesis is a good fit for the data. Given P1's action sequences, the state sequences, and the MDP $\tilde{\mathcal{H}}$ from Def. 11 the predicted P2's policy, the likelihood of P2's action sequences is computed by

$$
L_{0}=L\left(a_{2,0} a_{2,1} \ldots a_{2, n-1} \mid \pi_{2}^{*, 2}\right)=\prod_{i=0}^{n-1} \pi_{2}^{*, 2}\left(a_{2, i} \mid x_{i}, s_{i}\right),
$$

where $s_{i}=\tilde{v}_{i}[1]$ and $x_{i}=\tilde{v}_{i}[4]$ are the first and last components of $\tilde{v}_{i}$. 
At the same time, we obtain an estimate of P2's strategy from history as

$$
\hat{\pi}_{2}\left(a_{2} \mid \tilde{v}\right)= \begin{cases}\frac{\# \mathbf{1}\left(\tilde{v}, a_{2}\right)}{\# \mathbf{1}(\tilde{v})} & \text { if } \# \mathbf{1}(\tilde{v}) \neq 0, \\ \uparrow & \text { undefined otherwise. }\end{cases}
$$

where $\# \mathbf{1}\left(\tilde{v}, a_{2}\right)$ is the number of times $\mathrm{P} 2$ selects action $a_{2}$ given the current state $\tilde{v}$, and $\# \mathbf{1}(\tilde{v})$ is the number of times the state $\tilde{v}$ is visited. For unseen state-action pairs, we do not estimate the policy given the pair as it won't be used in the test. Based on the maximum likelihood estimate of P2's strategy from history, we have

$$
L_{1}=L\left(a_{2,0} a_{2,1} \ldots a_{2, n-1} \mid \hat{\pi}_{2}\right)=\prod_{i=0}^{n-1} \hat{\pi}_{2}\left(a_{2, i} \mid x_{i}, s_{i}\right) .
$$

The likelihood ratio is computed as

$$
\lambda=L_{0} / L_{1} .
$$

We conduct a likelihood ratio test and calculate $\chi_{n}^{2}=-2 \ln \lambda$, which is an approximate Chi-square distribution of $n$ degree of freedom, and $n$ is the number of parameters $\left\{\hat{\pi}_{2}\left(a_{2} \mid\right.\right.$ $\left.\tilde{v}) \mid \# \mathbf{1}\left(\tilde{v}, a_{2}\right) \neq 0\right\}$ estimated with maximum likelihood estimation. By selecting a confidence level $\alpha$, we reject the null hypothesis $H_{0}$ if $\chi_{n}^{2}$ is larger than the Chi-square percentile with $n$ degrees of freedom given the level $\alpha$.

\section{CASE STUdY}

In this section, we present an example to illustrate how this solution concept of dynamic level-2 hypergames gives rise to deceptive strategies for a class of opponent models that satisfy Assumption 2. This case study includes an inference function for P2 (Sec. IV-A) based on sliding-window change detection, the construction of MDP $\tilde{\mathcal{H}}$ for planning the deceptive optimal strategy for P1, with application to robot motion planning with temporal logic objectives in an adversarial environment.

\section{A. Inference with sliding-window change detection}

We introduce a class of inference algorithms based on change detection in Markov chain (MC) [38]. Given P2's finite hypothesis space $X, \mathrm{P} 2$ can construct a set of games $\{\mathcal{G}(x) \mid x \in X\}$. For each game $\mathcal{G}(x)$, it is assumed that there is an unique equilibrium $\left\langle\pi_{1}^{*}(x), \pi_{2}^{*}(x)\right\rangle$ where $\pi_{i}^{*}$ : PrefPlays $\times X \rightarrow \mathcal{D}\left(A_{i}\right)$ is a mixed strategy for player $i$. This equilibrium induces, from the concurrent game graph $\mathcal{G}$, a probability measure $\operatorname{Pr}^{\left\langle\pi_{1}^{*}(x), \pi_{2}^{*}(x)\right\rangle}$ over histories in $\mathcal{G}$. For simplicity in notation, we denote $\operatorname{Pr}^{\left\langle\pi_{1}^{*}(x), \pi_{2}^{*}(x)\right\rangle}$ by $\operatorname{Pr}^{x}$.

When P2's current hypothesis is $x, \mathrm{P} 2$ can detect a change from $x$ to some $x^{\prime} \in X$ using a sliding-window change detection algorithm, based on the Cumulative SUM (CUSUM) statistic [39]. First, we are given a data point in forms of history (joint state-action sequence) $h=s_{0} a_{0} s_{1} \ldots s_{n}$ and a nominal model $x_{0}$. We denote the interval of a time window of size $m+1$ as $[k, k+m]$, and the history within this time window is $s_{k} a_{k} \ldots s_{k+m} a_{k+m} s_{k+m+1}$. Second, we denote the $i$-th observation of the transitions within the time window as $y_{i}=\left(a_{k+i-1}, s_{k+i}\right)$ for any $i>0$. When $i=0$, the 0 -th observation within the window is $y_{0}=\left(s_{k}\right)$. Intuitively, given a data and a nominal model $x_{0}$, the sliding-window change detection algorithm uses a subsequence of history over a time window and detects if a change has occurred in the model that generates the data during this time window. Specifically, for each hypothesis $x \in X$ and a nominal model $x_{0}$, the algorithm computes the log-likelihood ratio,

$$
R_{j}^{x}=\sum_{i=0}^{j} r_{i}^{x}
$$

where $r_{i}^{x}=\ln \frac{\operatorname{Pr}^{x}\left(y_{i}\right)}{\operatorname{Pr}^{x}\left(y_{i}\right)}$, and $\operatorname{Pr}^{x}\left(y_{i}\right)\left(\right.$ resp. $\left.\mathbf{P r}^{x_{0}}\left(y_{i}\right)\right)$ is the probability of observing the transition given the probability measure $\operatorname{Pr}^{x}\left(\right.$ resp. $\left.\operatorname{Pr}^{x_{0}}\right)$.

The change detection lies in the difference between the log likelihood ratio and its current minimum value. The CUSUM score is given by

$$
Z_{k}^{x}=R_{k}^{x}-\min _{1 \leq j \leq k} R_{j}^{x} .
$$

Recursively, the CUSUM score is updated for each hypothesis $x \in X$ as

$$
Z_{k}^{x}=\max \left\{0, Z_{k-1}^{x}+\ln \frac{\operatorname{Pr}^{x}\left(y_{k}\right)}{\operatorname{Pr}^{x_{0}}\left(y_{k}\right)}\right\},
$$

where $Z_{0}^{x}=0$.

A change is detected at time $t$ when the score of at least one model, say $Z_{k}^{x}$, exceeds a user-defined constant threshold $c>0$. Formally, the time of change is given by

$$
t=\min \left\{k \mid \exists x \in X, Z_{k}^{x} \geq c\right\} .
$$

Once a change is detected, the algorithm sets the nominal model to be the current predicted model, disregards the history until the change, and keeps running the online change detection given new observation from the change point onwards. In the case when multiple models maintain similar CUSUM scores, we select one model based on some domain-specific heuristics or at uniformly random.

Lemma IV.1. Given a sliding-window change detection inference $\eta: X \times$ PrefPlays $\rightarrow X$ with window size $m+1$ and a finite hypothesis space $X$, two histories $h_{1}, h_{2}$ are $(\eta, x)$ equivalent if they share the same suffix 1 of length $m+1$.

The proof is based on the property of the change detection and thus omitted.

\section{B. Deceptive Planing with Temporal Logic Objective}

We consider two examples inspired by security games,

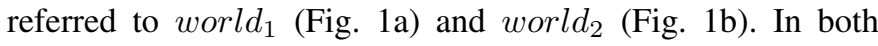
worlds, a robot (P1) is to visit several regions of interests (labelled $A, B, C$ and colored in red) according to a temporal ordering, and an observer (P2) can reallocate traps in cells colored in green. Both games are concurrent: When P1 selects an action to move, the P2 selects an action to set traps. When $\mathrm{P} 1$ enters the cell where P2 allocates the trap to that cell, P1 is trapped. The game terminates in two ways: a) P1 is trapped; b) P1 completes its task.

\footnotetext{
${ }^{1}$ For a word $w=\sigma_{1} \sigma_{2} \ldots \sigma_{n}$, a suffix of $w$ is a word $v$ of the form $\sigma_{i} \sigma_{i+1} \ldots \sigma_{n}$, where $1 \leq i \leq n+1$.
} 


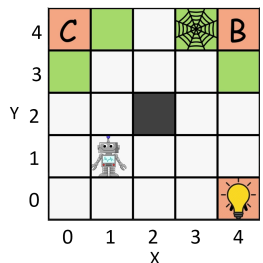

(a)

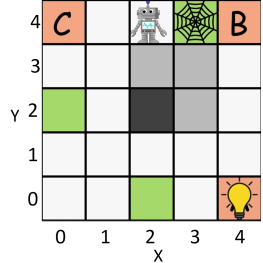

(b)

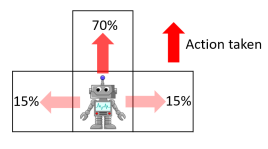

(c)
Fig. 1: (a): world 1 's initial configuration for P1 and P2. (b): world $_{2}$ 's initial configuration for P1 and P2. Cells colored in grey are walls. Bulb indicates initial P2's prediction. (c): Robot's dynamics when action "up" is taken.

Formally, we describe P1's task by the formula

$$
\left.\varphi_{1}=(\neg \mathrm{obs} \mathrm{U} A) \wedge(\neg(B \vee \mathrm{obs}) \mathrm{U} C)\right) .
$$

That is, the robot needs to visit $A$ and $C$ without reaching obstacles. Before visiting $C$, the robot cannot visit $B$. The corresponding DFA is drawn in Fig. 2.

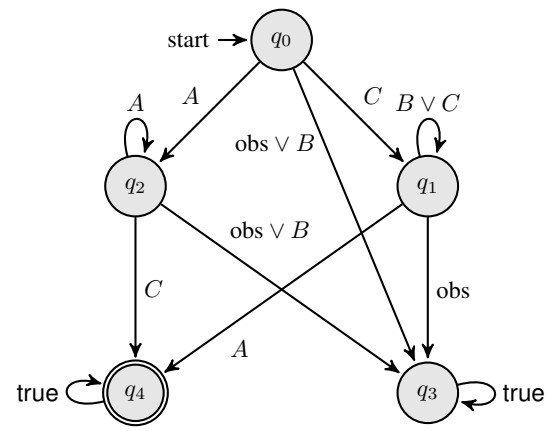

Fig. 2: The task automaton with 5 states and 12 edges corresponds to $\varphi_{1}$, where $Q=\left\{q_{i} \mid i=0,1,2,3,4\right\}$.

P1 can move in four compass directions, and P1's dynamics is plotted in Fig. 1c The grid world is surrounded with bouncing wall, i.e., if $\mathrm{P} 1$ hits the wall, it bounces back to its previous cell. The black cells in the grid world is a static obstacle, labeled by obs.

P2 can reallocate the traps (i.e., dynamic obstacles) to a subset of cells colored in green in world $d_{1}$ and world $_{2}$. P2 can only use $\ell$ traps with $n$ possible trap locations. Thus, the number of actions for the P2 is $\left(\begin{array}{l}n \\ \ell\end{array}\right)$, i.e., choose $\ell$ out of $n$. Every time $\mathrm{P} 2$ resets the location of any trap, it must wait at least $k$ time steps to be able to reallocate any trap again. In the example of $\operatorname{world}_{1}$, we select $n=4, \ell=1$, and let $k=0$; In the example of world $_{2}$, we select $n=3, \ell=1$, and let $k$ to be a variable.

In both examples: world $_{1}$ and world $_{2}$, the asymmetrical information is as follows:

- P1 knows the complete task $\varphi_{1}$.

- P2 does not know the complete task $\varphi_{1}$.

We refer to this situation as asymmetric information case. On the other side, if P2 knows P1's complete task, then we refer to that as symmetric information case. In the asymmetric information case, $\mathrm{P} 2$ has a hypothesis space $X=\{\neg \mathrm{obs} \mathrm{U} \phi \mid$ $\phi \in\{A, B, C\}\}$.
1) Different behaviors under asymmetric and symmetric information in world w $_{1}$ We compare P1's task completion rate between asymmetric information case and symmetric information case.

In asymmetric information case, for each $x \in X$, P2 solves a Stackelberg/leader-follower game and decides a trap configuration against the best response of $\mathrm{P} 1$ in game $\mathcal{G}(x)$. Let $A_{2}$ be the set of different configurations of traps. The strategy of $\mathrm{P} 2$ is to maximize the following objective function

$$
\pi_{2}^{B, 2}(s)=\arg \min _{a_{2} \in A_{2}} \max _{\pi_{1}} \operatorname{Pr}^{\pi_{1}}\left(h \models x \mid s, a_{2}\right) \text {, for all } s \in S,
$$

where $\operatorname{Pr}^{\pi_{1}}\left(h \models x \mid a_{2}\right)$ is the probability of P1 satisfying the formula $x$ given P2's action (trap configuration) $a_{2}$. For instance, if $x=\neg \mathrm{obs} \cup B$ and robot is at the $(2,4)$, then P2's optimal action is to allocates the trap to the green cell right to robot, that is $(3,4)$ in Fig. $1 \mathrm{a}$. For each hypothesis $x \in X$ and state $s \in S$, P2 solves the optimal trap allocation action $a_{2}$ and also computes the best response of $\mathrm{P} 1, \pi_{1}$, that achieves the maximum probability of satisfying $x$ from $s$. The joint strategy profile provides the probability measure $\mathbf{P r}^{x}$ for $\mathrm{P} 2$ to infer the subgoal of P1 using the sliding window change detection.

For the configuration of world $_{1}$, we evaluate different window sizes and find sliding-window size $m+1=2$ achieves a good trade-off in space complexity and accuracy in prediction. In case we choose an unsuitable window size, our method in subsection III-D enables P1 to detect the incorrect prediction of P2's behavior model caused by unsuitable windows size and increase the window size. Assuming P2 can reallocate traps anytime with $\pi_{2}^{B, 2}$, we can construct an MDP $\tilde{\mathcal{H}}$ and solve for P1's optimal deceptive strategy denoted by $\pi_{1}^{*}$.

In symmetric information case, $\mathrm{P} 1$ and $\mathrm{P} 2$ both have exact knowledge of task specification $\varphi_{1}$, and P1 wants to maximize the P1's probability of finishing the task; $\mathrm{P} 2$ wants to minimize the P1's probability of finishing the task. We denote the Nash Equilibrium strategy profile by $\left\langle\pi_{1}^{\dagger}, \pi_{2}^{\dagger}\right\rangle$. Where the Nash Equilibrium strategy profile is to solve the following objective function

$$
\left\langle\pi_{1}^{\dagger}, \pi_{2}^{\dagger}\right\rangle=\arg \min _{\pi_{2} \in \Pi_{2}} \max _{\pi_{1} \in \Pi_{1}} \operatorname{Pr}^{\pi_{1}, \pi_{2}}\left(h \models \varphi_{1}\right) .
$$

TABLE I: For world $_{1}$, completion rates for P1 in asymmetric information case and symmetric information case.

\begin{tabular}{lllr}
\hline Info & P1 Policy & P2 Policy & Completion rate (P1) \\
\hline Asymmetric & $\pi_{1}^{*}$ & $\pi_{2}^{B, 2}$ & $\mathbf{9 7 . 2 3 \%}$ \\
Symmetric & $\pi_{1}^{\dagger}$ & $\pi_{2}^{\dagger}$ & $33.55 \%$ \\
\hline
\end{tabular}

In Table I. we list two P1's completion rates of its task specification: one for asymmetric information case and one for symmetric information case case. From the Table [1] it indicates that under asymmetrical information, by following the deceptive strategy given P2 plays BSR strategy, P1 has much higher probability of satisfying the specification than that of the case by following the Nash equilibrium strategy profile. The deceptive strategy leverages this information asymmetry to lead P1 to achieve higher probability of finishing 
it's specification. We provide a short video ${ }^{2}$ to demonstrate the difference on P2's behaviors in asymmetric information case and symmetric information case.

Next, we want to understand whether a delay in reallocating traps for P2 would effect the completion rate of P1. However, in the world $_{1}$ example, we observed in experiments that any delay on reallocation could easily lead P1 to complete its task. Based on these observations, we construct another example world $_{2}$, and evaluate the complete rates for every $k$ steps of delay and correctness of model mismatch in this example world $_{2}$.

2) Reallocation every $k$ steps of delay in world w $_{2}$ In this example, we assume that P2 is restricted to only reallocate the trap after $k$ steps since the last reallocation, where $k$ is an integer. P1 is aware of P2's delay $k$ and synthesize the deceptive strategy. Figure 3 shows the completion rate of task (values of in initial state $(2,4)$ in Fig. 1b) under different steps of delay up to $k=3$. The results indicates that with the increase of steps of delay, the probability of completing the task increases and P1 exploits P2's delay and lack of information.

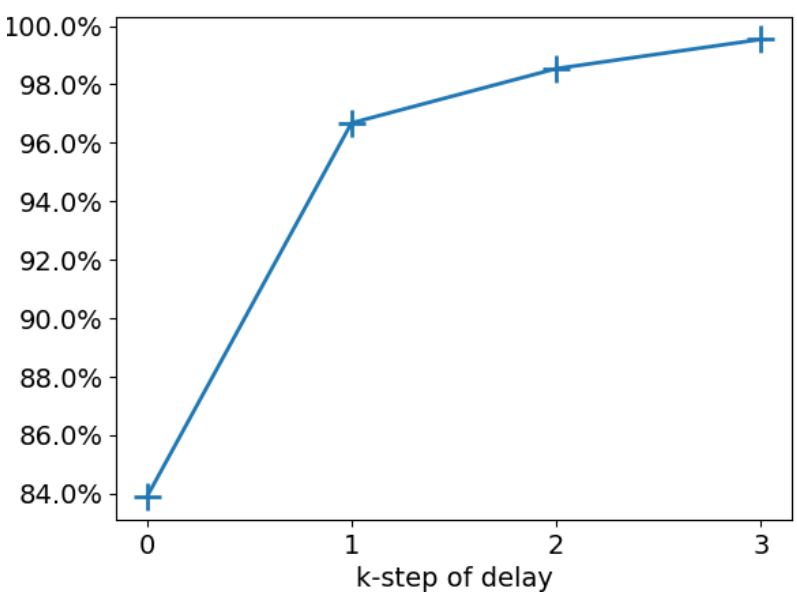

Fig. 3: The task completion rates of $\mathrm{P} 1$ given $\mathrm{P} 2$ with $k$-step delay in reallocating traps, for $k=0,1,2,3$.

3) Detection of Model mismatch in world ${ }_{2}$ : We use experiments in the configuration world $_{2}$ to demonstrate the correctness of the detect mechanism, that is, to identify whether there is a deviation from the predicted opponent modeling of P2. We set the significance level $\alpha=0.05$. If the likelihood of observed action sequences is smaller than or equal to 0.05 , we reject the null hypothesis: the data is generated by our predicted model of $\mathrm{P} 2$.

We consider a case that P1 follows policy $\pi_{1}^{*}$, and P2 plays her policy predicted by P1 for first four steps. After first four steps, we let P2 plays a random policy $\pi_{2}^{R}$, i.e., $\pi_{2}^{R}(a \mid s)=$ $\frac{1}{|A(s)|}$, for all $a \in A(s)$. The mismatch is detected at the 7th step of the online interaction, and P1 is alerted that P2 deviates from the predicted policy. We compute $\lambda$ after each step and plot it in Fig. 4, where we also plot the $\chi^{2}$. (The

2 https://www.dropbox.com/s/7xxrq6mzm9umvva/07162020.mpeg?dl=0 reason predicted $\lambda=0$ is because the predicted policy $\pi_{2}^{B, 2}$ is deterministic.) From Fig. 4, we see that at the 7-th step of online interaction, we have $\lambda>\chi^{2}$, so we reject the null hypothesis. The degree of freedom in the Chi-square detector is the number of the state-action pairs.

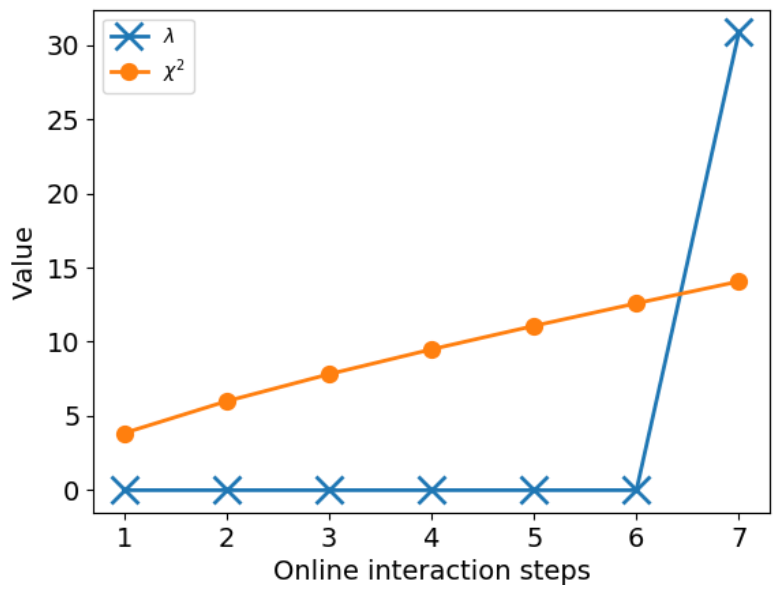

Fig. 4: The likelihood ratio $\lambda$ for online interaction between $\mathrm{P} 1$ and $\mathrm{P} 2$.

\section{CONClusion}

In this work, we propose a solution concept for a class of hypergames to solve deceptive strategies with temporal logic objectives. Our hypergame framework identifies two key components for deceptive planning: Opponent modeling and proactive planning. The general framework can be extended to other class of games with incomplete information. It is also important to note that the proposed approach does not generalize easily to partially observable games with two-sided partial observations. This is because that two players may have different observations over the same history and incomplete information about what observation the other player has. The partial observation may potentially make player 1's subjective model of player 2's perceptual game diverges from the actual perceptual game of player 2 .

Building on this work, our future work will focus on adaptive and robust deception for defense. In the class of hypergames considered, we have made some assumptions about the inference mechanism and strategies employed by P2. To generalize from deterministic inference to probabilistic inference, the Markov decision process reduced from the game can be continuous, because the hypothesis space $X$ is probabilistic distributions. In addition, robust Markov decision processes can be incorporated to deal with mismatches in the opponent model, provided the range of mismatches is known a prior. Robust and adaptive deception is also needed to deal with the case when P2 may have multiple BSR strategy, as P1 needs to learn which strategy P2 uses and adapts its deceptive strategy accordingly. We will also consider practical applications of the deceptive planning for security applications in cyber-physical systems. 


\section{REFERENCES}

[1] U. Gneezy, "Deception: The role of consequences," The American Economic Review, vol. 95, no. 1, p. 384, 2005.

[2] M. I. Handel, Masters of war: classical strategic thought. Routledge, 2005.

[3] F. J. Stech, K. E. Heckman, and B. E. Strom, "Integrating cyber-d\&d into adversary modeling for active cyber defense," in Cyber Deception, 2016.

[4] K. Horák, Q. Zhu, and B. Bošanskỳ, "Manipulating adversarys belief: A dynamic game approach to deception by design for proactive network security," in International Conference on Decision and Game Theory for Security. Springer, 2017, pp. 273-294.

[5] L. Huang and Q. Zhu, "Dynamic bayesian games for adversarial and defensive cyber deception," ArXiv, vol. abs/1809.02013, 2018.

[6] M. Ahmadi, M. Cubuktepe, N. Jansen, S. Junges, J.-P. Katoen, and U. Topcu, "The partially observable games we play for cyber deception," ArXiv, vol. abs/1810.00092, 2018.

[7] A. R. Wagner and R. C. Arkin, "Acting deceptively: Providing robots with the capacity for deception," International Journal of Social Robotics, vol. 3, no. 1, pp. 5-26, 2011.

[8] M. Egorov, M. J. Kochenderfer, and J. J. Uudmae, "Target surveillance in adversarial environments using pomdps," in Proceedings of the Thirtieth AAAI Conference on Artificial Intelligence. AAAI Press, 2016, pp. 2473-2479.

[9] P. Masters and S. Sardina, "Deceptive path-planning," in Proceedings of the 26th International Joint Conference on Artificial Intelligence. AAAI Press, 2017, pp. 4368-4375.

[10] B. Gharesifard and J. Cortes, "Evolution of players' misperceptions in hypergames under perfect observations," IEEE Transactions on Automatic Control, vol. 7, no. 57, pp. 1627-1640, 2012.

[11] M. Ornik and U. Topcu, "Deception in optimal control," in 2018 56th Annual Allerton Conference on Communication, Control, and Computing (Allerton). IEEE, 2018, pp. 821-828.

[12] Z. Manna and A. Pnueli, The Temporal Logic of Reactive and Concurrent Systems. Berlin, Heidelberg: Springer-Verlag, 1992.

[13] O. Kupferman and M. Y. Vardi, "Model checking of safety properties," in $C A V, 1999$

[14] T. Zhang, L. Huang, J. Pawlick, and Q. Zhu, "Game-theoretic analysis of cyber deception: Evidence-based strategies and dynamic risk mitigation," ArXiv, vol. abs/1902.03925, 2019.

[15] J. T. House and G. Cybenko, "Hypergame theory applied to cyber attack and defense," in Sensors, and Command, Control, Communications, and Intelligence (C3I) Technologies for Homeland Security and Homeland Defense IX, E. M. Carapezza, Ed., vol. 7666, International Society for Optics and Photonics. SPIE, 2010, pp. $39-49$.

[16] K. Ferguson-Walter, S. Fugate, J. Mauger, and M. Major, "Game theory for adaptive defensive cyber deception," in Proceedings of the 6th Annual Symposium on Hot Topics in the Science of Security, ser. HotSoS '19. New York, NY, USA: ACM, 2019, pp. 4:1-4:8.

[17] M. Wang, K. W. Hipel, and N. M. Fraser, "Solution concepts in hypergames," Applied Mathematics and Computation, vol. 34, no. 3, pp. 147 - 171, 1989.

[18] P. G. Bennett, "Hypergames: developing a model of conflict," Futures, vol. 12 , no. 6, pp. 489-507, 1980.

[19] B. Gharesifard and J. Cortés, "Stealthy deception in hypergames under informational asymmetry," IEEE Transactions on Systems, Man, and Cybernetics: Systems, vol. 44, no. 6, pp. 785-795, 2014.

[20] M. O. Sayin and T. Basar, "Deception-as-defense framework for cyberphysical systems," arXiv preprint arXiv:1902.01364, 2019.

[21] R. M. Góes, E. Kang, R. Kwong, and S. Lafortune, "Stealthy deception attacks for cyber-physical systems," in 2017 IEEE 56th Annual Conference on Decision and Control (CDC). IEEE, 2017, pp. 4224-4230.

[22] K. Chatterjee and T. A. Henzinger, "A survey of stochastic $\omega$-regular games," Journal of Computer and System Sciences, vol. 78, no. 2, pp. 394-413, 2012.

[23] R. Bloem, K. Chatterjee, and B. Jobstmann, "Graph Games and Reactive Synthesis," in Handbook of Model Checking, E. M. Clarke, T. A. Henzinger, H. Veith, and R. Bloem, Eds. Cham: Springer International Publishing, 2018, pp. 921-962.

[24] L. de Alfaro, T. A. Henzinger, and O. Kupferman, "Concurrent reachability games," Theoretical Computer Science, vol. 386, no. 3, pp. 188 217, Nov. 2007.

[25] N. Piterman, A. Pnueli, and Y. Sa' ar, "Synthesis of Reactive(1) Designs," in Verification, Model Checking, and Abstract Interpretation, ser. Lecture Notes in Computer Science, E. A. Emerson and K. S. Namjoshi, Eds. Berlin, Heidelberg: Springer, 2006, pp. 364-380.
[26] J. Klein and C. Baier, "Experiments with deterministic $\omega$-automata for formulas of linear temporal logic," Theoretical Computer Science, vol. 363, no. 2, pp. 182-195, 2006.

[27] A. Pnueli and R. Rosner, "On the synthesis of a reactive module," 1989 , pp. $179-190$.

[28] M. J. Osborne and A. Rubinstein, A course in game theory, 1994.

[29] R. R. I. Vane, "Using Hypergames to Select Plans in Competitive Environments," Ph.D. dissertation, George Mason University, 2000.

[30] Y. Sasaki and K. Kijima, "Hierarchical hypergames and bayesian games: A generalization of the theoretical comparison of hypergames and bayesian games considering hierarchy of perceptions," Journal of Systems Science and Complexity, vol. 29, no. 1, pp. 187-201, 2016.

[31] L. Huang and Q. Zhu, "A dynamic games approach to proactive defense strategies against advanced persistent threats in cyber-physical systems," Computers \& Security, vol. 89, p. 101660, 2020.

[32] E. F. Moore et al., "Gedanken-experiments on sequential machines," Automata studies, vol. 34, pp. 129-153, 1956.

[33] C. Baier and J.-P. Katoen, Principles Of Model Checking, 2008, vol. 950.

[34] M. Kwiatkowska, G. Norman, and D. Parker, "Stochastic model checking," in Formal Methods for the Design of Computer, Communication and Software Systems: Performance Evaluation (SFM'07), ser. LNCS (Tutorial Volume), M. Bernardo and J. Hillston, Eds., vol. 4486. Springer, 2007, pp. 220-270.

[35] — "PRISM 4.0: Verification of probabilistic real-time systems," in Proc. 23rd International Conference on Computer Aided Verification ( $\left.C A V^{\prime} 11\right)$, ser. LNCS, G. Gopalakrishnan and S. Qadeer, Eds., vol. 6806. Springer, 2011, pp. 585-591.

[36] L. Li and J. Fu, "Topological approximate dynamic programming under temporal logic constraints," in 2019 IEEE 58th Conference on Decision and Control (CDC), Dec 2019, pp. 5330-5337.

[37] T. A. Severini, Likelihood methods in statistics. Oxford University Press, 2000.

[38] A. M. Polansky, "Detecting change-points in Markov chains," Computational Statistics and Data Analysis, vol. 51, no. 12, pp. 6013-6026, 2007.

[39] M. Basseville, I. V. Nikiforov et al., Detection of abrupt changes: theory and application. Prentice Hall Englewood Cliffs, 1993, vol. 104.

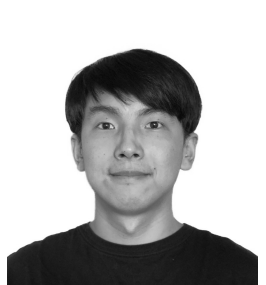

Lening Li (Student Member, IEEE) received a B.S. degree in Information Security (Computer Science) from Harbin Institute of Technology, China in 2014, and an M.S. degree in Computer Science from Worcester Polytechnic Institute, Worcester, MA, USA, in 2016. He is currently pursuing a $\mathrm{Ph} . \mathrm{D}$. degree in Robotics Engineering at Worcester Polytechnic Institute, Worcester, MA, USA.

His research interests include reinforcement learning, stochastic control, game theory, and formal methods.

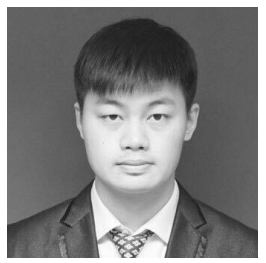

Haoxiang Ma (Student Member, IEEE) received the B.S. degree in Computer Science from the Nankai University, China in 2017, and the M.S. degree in Computer Science from Worcester Polytechnic Institute, Worcester, MA, USA, in 2019. He is currently pursuing the Ph.D. degree in Robotics Engineering at Worcester Polytechnic Institute, Worcester, MA, USA.

His interests include: Reinforcement Learning, Optimal control, Game theory, with applications to robotic systems. 


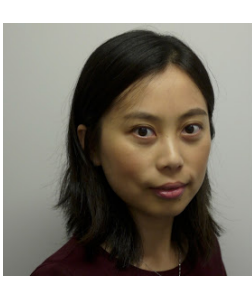

Jie Fu (Member, IEEE) received the B.S. and M.S. degrees from Beijing Institute of Technology, Beijing, China, in 2007 and 2009, respectively, and the Ph.D. degree in mechanical engineering from the University of Delaware in 2013. She is currently an Assistant Professor at the Department of Electrical and Computer Engineering, Robotics Engineering Program, Worcester Polytechnic Institute.

Her research interests include probabilistic and formal methods.

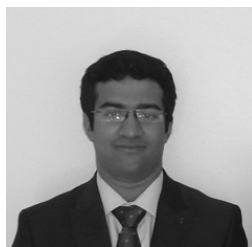

Abhishek N. Kulkarni (Student Member, IEEE) received the B.Tech. degree in Electronics and Telecommunications Engineering from the Vishwakarma Institute of Technology, India in 2016. $\mathrm{He}$ is currently pursuing his Ph.D. degree in Robotics Engineering at Worcester Polytechnic Institute, Worcester, MA, USA.

His interests include: game and hypergame theory, formal methods with applications to robotic systems and cybersecurity. 